\title{
16 UK Balance of payments
}

\subsection{Batance of payments \\ Summary}

$£$ million

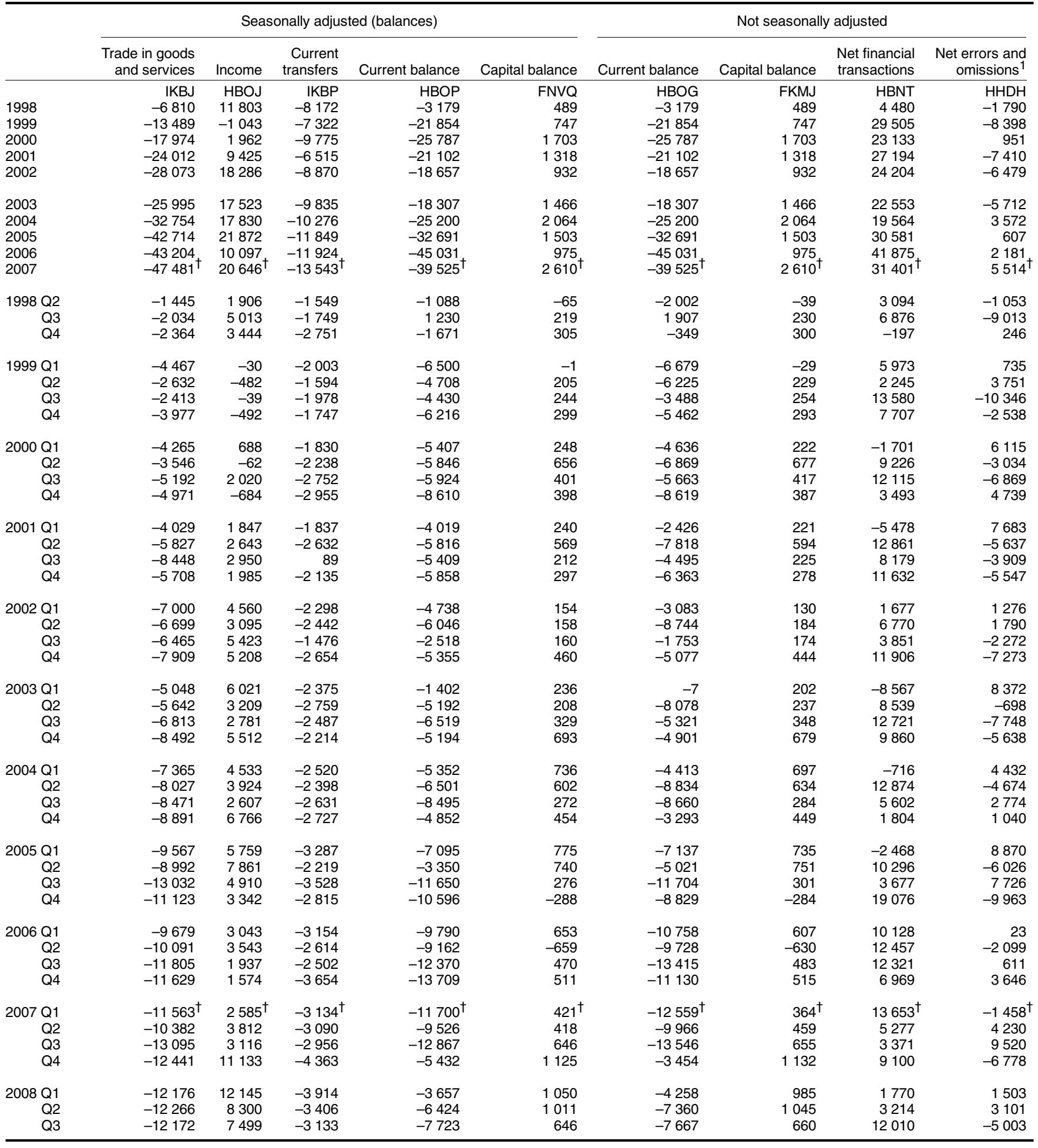

1 This series represents net errors and omissions in the balance of payments accounts. It is the converse of the current and capital balances (HBOG and FKMJ) and net financial account transactions (HBNT) and is required to bal-

Source: Office for National Statistics: 02070142002 ance these three accounts, not seasonally adjusted. 


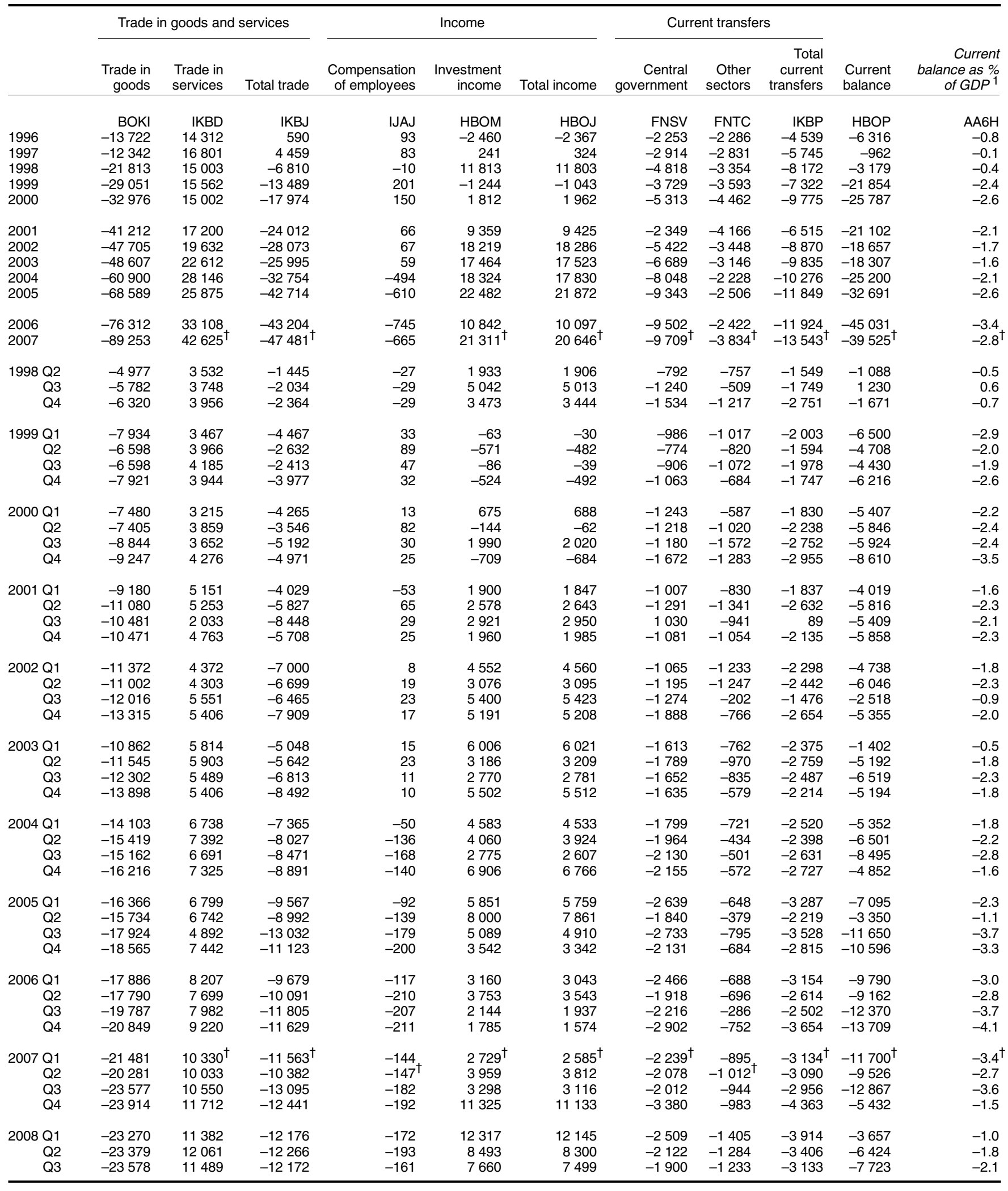




\begin{tabular}{|c|c|c|c|c|c|c|c|c|c|c|c|c|c|c|c|}
\hline & \multicolumn{3}{|c|}{ Investment in the UK } & \multicolumn{6}{|c|}{ UK investment abroad } & \multicolumn{5}{|c|}{ Net transactions } & \multirow[b]{3}{*}{ Total } \\
\hline & \multirow{2}{*}{$\begin{array}{r}\text { Direct } \\
\text { investme- } \\
\mathrm{nt}\end{array}$} & \multirow{2}{*}{\multicolumn{2}{|c|}{$\begin{array}{c}\text { Portfolio } \\
\text { Other } \\
\text { investme- investme- } \\
\text { nt }\end{array}$}} & \multirow[b]{2}{*}{ Total } & \multirow{2}{*}{$\begin{array}{r}\text { Direct } \\
\text { investme- i } \\
\mathrm{nt}\end{array}$} & \multirow{2}{*}{$\begin{array}{r}\text { Portfolio } \\
\text { investme- } \\
\text { nt }\end{array}$} & \multirow{2}{*}{$\begin{array}{c}\text { Financial } \\
\text { derivati- i } \\
\text { ves }\end{array}$} & \multicolumn{2}{|c|}{$\begin{array}{l}\text { Other } \\
\text { investme- Reserve }\end{array}$} & \multirow{2}{*}{\multicolumn{2}{|c|}{$\begin{array}{c}\text { Direct } \\
\text { investme- ir } \\
\mathrm{nt}\end{array}$}} & \multirow{2}{*}{$\begin{array}{r}\text { Portfolio } \\
\text { investme- } \mathrm{i} \\
\mathrm{nt}\end{array}$} & $\begin{array}{l}\text { Other } \\
\text { ivestme- R }\end{array}$ & Reserve & \\
\hline & & & & & & & & nt & assets & & & & nt & assets & \\
\hline & HJYU & $\mathrm{HHZF}$ & XBMN & HBNS & -HJYP & $-\mathrm{HHZC}$ & -ZPNN & -XBMM & -LTCV & -HBNR & HJYV & HHZD & HHYR & LTCV & HBNT \\
\hline 1998 & 45054 & 25038 & 73117 & 143209 & 73786 & 32072 & 3043 & 29992 & -164 & 138729 & -28732 & -7034 & 43125 & 164 & 4480 \\
\hline 1999 & 55066 & 106339 & 53312 & 214717 & 125602 & 21389 & -2685 & 41545 & -639 & 185212 & -70536 & 84950 & 11767 & 639 & 29505 \\
\hline 2000 & 80566 & 172177 & 235563 & 488306 & 155582 & 65563 & -1553 & 241666 & 3915 & 465173 & -75016 & 106614 & -6103 & -3915 & 23133 \\
\hline 2001 & 37348 & 40829 & 237558 & 315735 & 42827 & 86553 & -8417 & 170663 & -3085 & 288541 & -5479 & -45724 & 66895 & 3085 & 27194 \\
\hline 2002 & 16782 & 49740 & 62648 & 129170 & 35041 & 1010 & -1001 & 70375 & -459 & 104966 & -18259 & 48730 & -7727 & 459 & 24204 \\
\hline 2003 & 16776 & 105648 & 241529 & 363953 & 40889 & 36268 & 5401 & 01 & -1559 & 41400 & -24113 & 69380 & -18872 & 1559 & 22553 \\
\hline 2004 & 31208 & 88549 & 425577 & 545334 & 51510 & 140850 & 7875 & 325339 & 196 & 525770 & -20302 & -52301 & 100238 & -196 & 19564 \\
\hline 2005 & 97845 & 132718 & 487400 & 717963 & 44005 & 151039 & -9556 & 501238 & 656 & 687382 & 53840 & -18321 & -13838 & -656 & 30581 \\
\hline 2006 & 80539 & 152609 & 382315 & 615463 & 47155 & 138588 & -7449 & 395720 & -426 & 573588 & + 33384 & 14021 & -13405 & 426 & 41875 \\
\hline 2007 & $98150^{\dagger}$ & $207791^{\dagger}$ & $720868^{\dagger_{1}}$ & $t_{1} 026809^{\dagger}$ & $136130^{\dagger}$ & $92015^{\dagger}$ & 19001 & $747071^{\dagger}$ & 1191 & $995408^{\dagger}$ & $\dagger-37980^{\dagger}$ & $115776^{\dagger}$ & $-26203^{\dagger}$ & -1191 & $31401^{\dagger}$ \\
\hline 1998 Q2 & 8055 & -8470 & 57050 & 56635 & 7107 & 9159 & 595 & 36371 & 309 & 53541 & 948 & -17629 & 20679 & -309 & 3094 \\
\hline 1999 Q1 & 12832 & 25 & 02 & 359 & & & 19 & & -837 & 386 & 83 & 447 & 13 & 837 & 5973 \\
\hline Q2 & 5262 & 82988 & 71 & 138121 & $\$ 1$ & 12 & 441 & & 202 & 376 & -79 & 70881 & & -202 & 2245 \\
\hline Q3 & 12863 & 4920 & -39504 & -21721 & 11589 & 9964 & 535 & -56 & -759 & -3 & 1274 & -5044 & 17126 & 759 & 13580 \\
\hline Q4 & 24109 & 2606 & -35757 & -9042 & 21203 & -16060 & -2142 & -20505 & 755 & -16749 & 2906 & 18666 & -15252 & -755 & 7707 \\
\hline 2000 Q1 & 14601 & 100491 & 112672 & 227764 & 112 & -13178 & 492 & 131 & -2465 & 229465 & -98390 & 69 & -18953 & 2465 & -1701 \\
\hline Q2 & 22735 & 30323 & 61731 & 114789 & 34299 & 45176 & -926 & 26468 & 546 & 105563 & -11564 & -14853 & 35263 & -546 & 9226 \\
\hline Q3 & 43931 & 11985 & 33048 & 88964 & 5849 & 19520 & -526 & 50476 & 1530 & 76849 & 38082 & -7535 & -17428 & -1530 & 12115 \\
\hline Q4 & -701 & 29378 & 28112 & 56789 & 2443 & 14045 & -593 & 33097 & 4304 & 53296 & -3144 & 15333 & -4985 & -4304 & 3493 \\
\hline 2001 Q1 & 16426 & & & 711 & & & -2331 & & 599 & 189 & -4730 & -1 & & -399 & -5478 \\
\hline Q2 & 12210 & & -20 & -2611 & 11835 & & 1473 & $-5 \varepsilon$ & 37 & -15472 & 375 & -24618 & 38614 & -37 & 12861 \\
\hline Q3 & 5518 & 7175 & -10 & 2390 & 8648 & 11047 & -5843 & -19143 & -498 & -5789 & -3130 & -3872 & 8840 & 498 & 8179 \\
\hline Q4 & 3194 & 8531 & 56520 & 68245 & 1188 & 8123 & -1716 & 49043 & -25 & 56613 & 2006 & 408 & 7477 & 25 & 11632 \\
\hline 2002 Q1 & -6050 & 14 & 38025 & 389 & & -6577 & -340 & 29 & -528 & 12 & -24 & 91 & 9 & 528 & 1677 \\
\hline Q2 & 15376 & 21646 & 1504 & 38526 & 16315 & 42400 & -1968 & -25013 & 22 & 31756 & -939 & -20754 & 26517 & -22 & 6770 \\
\hline Q3 & 1061 & 2343 & -1739 & 1665 & 15331 & -36797 & 1855 & 16743 & 682 & -2186 & -14270 & 39140 & -18482 & -682 & 3851 \\
\hline $\mathrm{Q}$ & 5771 & 14687 & 107687 & 128145 & 1 & 25 & -23 & 82 & -647 & 119606 & -8442 & -10740 & 24772 & 647 & 8539 \\
\hline Q3 & 1186 & 44208 & 14616 & 60010 & 73 & -3958 & 1348 & 41273 & 1303 & 47289 & -6137 & 48166 & -26657 & -1303 & 12721 \\
\hline Q4 & 6163 & 28864 & -9657 & 25370 & -1641 & -727 & -1322 & 19789 & -589 & 15510 & 7804 & 29591 & -29446 & 589 & 9860 \\
\hline 2004 Q1 & 5272 & 53168 & 219446 & 277886 & & 5902 & 4504 & 212177 & -525 & $27 \varepsilon$ & -11 & 7 & 7269 & 525 & -716 \\
\hline Q2 & 6038 & 13225 & 65824 & 85087 & 12857 & -198 & 3967 & 56182 & -595 & 72213 & -6819 & 13423 & 9642 & 595 & 12874 \\
\hline Q3 & 7360 & 15665 & 84968 & 107993 & 19121 & 47649 & 1278 & 34397 & -54 & 102391 & -11761 & -31984 & 50571 & 54 & 5602 \\
\hline Q4 & 12538 & 6491 & 55339 & 74368 & 2988 & 47497 & -1874 & 22583 & 1370 & 72564 & 9550 & -41006 & 32756 & -1370 & 1804 \\
\hline 2005 Q1 & 1 & 5 & & & & 176 & 37 & & -533 & & & & & 533 & 68 \\
\hline Q2 & 14525 & 16 & & 066 & & & -373 & & 527 & 164770 & 12123 & -19782 & 18 & -527 & 10296 \\
\hline Q3 & 70710 & 4848 & 93865 & 169423 & 9738 & 49275 & -9211 & 115568 & 376 & 165746 & 60972 & -44427 & -21703 & -376 & 3677 \\
\hline Q4 & -3232 & 40253 & 62355 & 99376 & 9226 & 35399 & -3762 & 39151 & 286 & 80300 & -12458 & 4854 & 23204 & -286 & 19076 \\
\hline 2006 Q1 & 33131 & 27742 & 290389 & 351262 & 4851 & & 827 & 297042 & -465 & 341134 & & -11137 & & 465 & 10128 \\
\hline Q2 & 22010 & 22388 & -39954 & 4444 & -4582 & 24333 & -174 & -27380 & -210 & -8013 & 26592 & -1945 & -12574 & 210 & 12457 \\
\hline Q3 & 18762 & 61120 & 55629 & 135511 & 10626 & 9940 & 2101 & 100138 & 385 & 123190 & 8136 & 51180 & -44509 & -385 & 12321 \\
\hline Q4 & 6636 & 41359 & 76251 & 124246 & 36260 & 65436 & -10203 & 25920 & -136 & 117277 & -29624 & -24077 & 50331 & 136 & 6969 \\
\hline 2007 Q1 & $13656^{\dagger}$ & $34642^{\dagger}$ & $\dagger 410749^{\dagger}$ & ${ }^{\dagger} 459047^{\dagger}$ & $6616^{\dagger}$ & $62521^{\dagger}$ & 6057 & $371024^{\dagger}$ & -824 & $445394^{\dagger}$ & $7040^{\dagger}$ & $-27879^{\dagger}$ & $39725^{\dagger}$ & 824 & $13653^{\dagger}$ \\
\hline Q2 & 23921 & 92041 & 89290 & 205252 & 29824 & 51297 & 2522 & 116265 & 67 & 199975 & -5903 & 40744 & -26975 & -67 & 5277 \\
\hline Q3 & 13395 & 31870 & 117656 & 162921 & 22985 & -9809 & 3537 & 143395 & -558 & 159550 & -9590 & 41679 & -25739 & 558 & 3371 \\
\hline Q4 & 47178 & 49238 & 103173 & 199589 & 76705 & -11994 & 6885 & 116387 & 2506 & 190489 & -29527 & 61232 & -13214 & -2506 & 9100 \\
\hline 2008 Q1 & 26052 & 52541 & 217731 & 296324 & 19633 & 21013 & 29404 & 267461 & -931 & 294554 & 6419 & 73554 & -49730 & 931 & 1770 \\
\hline Q2 & 15998 & 105558 & -469461 & -347905 & 21045 & 1434 & $-10810^{\dagger}$ & -362896 & 108 & -351119 & -5047 & 104124 & -106565 & -108 & 3214 \\
\hline Q3 & 4462 & 51273 & 29055 & 84790 & 16167 & -2802 & -12469 & 74016 & 132 & 72780 & -11705 & 54075 & -44961 & 2132 & 12010 \\
\hline
\end{tabular}

